\title{
EQUAÇÕES DE VOLUME DE MADEIRA PARA O CERRADO DE PLANALTINA DE GOIÁS
}

\author{
José Imaña-Encinas ${ }^{1}$, Otacílio Antunes Santana ${ }^{2}$, José Elias de Paula ${ }^{3}$, Christian Rainier Imaña ${ }^{4}$ \\ ${ }^{1}$ Eng. Florestal, Ph.D., Depto. Engenharia Florestal, UnB, Brasília, DF, Brasil - imana@unb.br \\ ${ }^{2}$ Biólogo, Dr., Depto. Geografia, UnB, Brasília, DF, Brasil - otaciliosantana@gmail.com \\ ${ }^{3}$ Botânico, Dr., Depto. de Botânica, UnB, Brasília, DF, Brasil - depaula@unb.br \\ ${ }^{4}$ Estatístico, Sec. de Fazenda do Estado de Minas Gerais, Belo Horizonte, MG, Brasil - christian_bsb@ hotmail.com
}

Recebido para publicação: 05/10/2007 - Aceito para publicação: 03/07/2008

\begin{abstract}
Resumo
Foi estudado um hectare de cerrado stricto sensu situado em Planaltina, Distrito Federal, com o objetivo de testar equações volumétricas que permitam quantificar o volume de madeira existente nessa fitofisionomia. Foram instaladas 10 parcelas de 10 x $100 \mathrm{~m}$ na área de estudo. As variáveis dendrométricas consideradas na árvore em pé foram DAP e altura total. Na cubagem rigorosa, foram medidos os comprimentos (altura) do fuste e os diâmetros na altura de 0,30 $\mathrm{m}$ e na altura da primeira bifurcação do fuste. Nos galhos, foram medidos os diâmetros na base do galho e na altura da bifurcação e o comprimento entre esses diâmetros. Calcularam-se os volumes de madeira com casca dos fustes e dos galhos de cada indivíduo das espécies identificadas. Foram medidas 490 árvores com DAP igual ou superior a $5 \mathrm{~cm}$, que ficaram distribuídas em 51 espécies. Foram testados 18 modelos volumétricos. A cubagem rigorosa determinou um volume de madeira igual a $16,18 \mathrm{~m}^{3} / \mathrm{ha}$ com casca, correspondendo a 10,85 (67 \%) dos fustes e 5,33 (33\%) dos galhos. O modelo volumétrico que melhor se ajustou aos dados coletados foi o de Rezende et al. (2006), que apresentou o maior $\mathrm{R}_{\text {aj }}^{2}$ e menor desvio padrão $\left(S_{x y}\right)$ da estimativa.

Palavras-chave: Inventário florestal; cubagem rigorosa.
\end{abstract}

\begin{abstract}
Wood volume equation for a cerrado at Planaltina, Goiás State. A study was carried out on one hectare of the cerrado sensu stricto vegetation located at Planaltina, Federal District. Ten plots of 10 $\mathrm{x} 100 \mathrm{~m}$ each formed the survey area. Measurements of height, dbh and diameters at $0.20 \mathrm{~m}$ and at bifurcation height of the stem and the diameters and length of the branches were done. Descriptive wood volume parameters were obtained from 490 trees with dbh larger than $5.0 \mathrm{~cm}$, distributed within 51 species. The obtained dbh average was $10.25 \mathrm{~cm}$ and the basal area $4.924222 \mathrm{~m}^{2} / \mathrm{ha}$. The wood volume was $16.18 \mathrm{~m}^{3} /$ ha with bark corresponding to $10.85(67 \%)$ of the stem and $5.33(33 \%)$ of the branches. The volumetric model that highly adjusted to sampled data was the Rezende et al. Model. This model showed high $\mathrm{R}_{\text {aj }}^{2}$ and low standard deviation.

Keywords: Forest inventory; wood volume measurement.
\end{abstract}

\section{INTRODUÇÃO}

Na região do Distrito Federal e em áreas do Centro-Oeste, a vegetação natural apresenta diversas fitofisionomias de estruturas florestais, como as matas mesofiticas, as matas de galeria, o cerradão e o próprio cerrado. Fisionomicamente, considerando só o estrato arbóreo, especificamente o cerrado é constituído de um grande mosaico de comunidades vegetais arbóreas com um dossel bastante aberto até mais ou menos fechado, com presença de um estrato arbóreo-arbustivo acompanhado de uma camada rasteiro-graminosa contínua. O cerrado stricto sensu (cerrado s.s.) é caracterizado como uma vegetação arbórea esparsa de fustes tortuosos com alturas inferiores a 15 m (RATTER et al., 2003).

Diversos estudos florísticos e fitossociológicos foram realizados na região do cerrado (FELFILI et al., 2004; FELFILI; SILVA JÚNIOR, 2001; FONSECA; SILVA JÚNIOR, 2004), tendo fornecido importantes informações sobre a sua riqueza florística, o número de indivíduos por hectare e a área basal ocupada. A revisão bibliográfica encontrou poucas referências (SCOLFORO; SILVA, 1993; PAULA et 
al., 1998; OLIVEIRA et al., 1998; IMAÑA-ENCINAS; PAULA, 2003; BATALHA et al., 2004; REZENDE et al., 2006) relativas ao volume de madeira da fitofisionomia cerrado. Deduz-se, consequentemente, que o parâmetro volumétrico de madeira para as áreas de cerrado é pouco conhecido, mesmo reconhecendo a existência, em várias regiões com vegetação nativa, de uma intensiva extração madeireira para fins energéticos.

O presente trabalho teve como escopo avaliar equações volumétricas, e sua correspondente distribuição diamétrica, ajustadas às características das variáveis dendrométricas do cerrado, e assim fornecer subsídios paramétricos que possam ser incorporados em possíveis planos de manejo da vegetação natural dessa fitofisionomia.

\section{MATERIAL E MÉTODOS}

A área de estudo está localizada na região de Planaltina de Goiás, junto à divisa com o Distrito

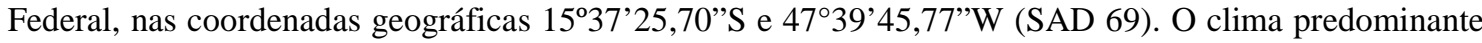
da região corresponde ao tipo Cwa da classificação de Köppen, com uma precipitação que varia entre 1.400 a $1.450 \mathrm{~mm} / \mathrm{ano}$. A declividade está entre 2 e $3 \%$, e a altitude próxima a $948 \mathrm{~m}$.

$\mathrm{O}$ hectare medido foi dividido em 10 parcelas retangulares de $10 \times 100 \mathrm{~m}$, que ficaram localizadas em forma contígua, uma ao lado da outra, perfazendo assim um hectare de observação. Coletou-se todo o material botânico fértil de todas as espécies inventariadas. O material botânico de cada espécie recebeu correspondente número de coleta, que após a secagem das exsicatas em estufa, foi incorporado ao acervo do Herbário da Universidade de Brasília (UB).

Todas as árvores com DAP igual ou superior a $5 \mathrm{~cm}$ foram consideradas, sendo realizadas nelas medidas de cubagem rigorosa. Em pé, foi medido o DAP e a altura total da árvore. No fuste das árvores, foram consideradas as seguintes variáveis: DAP, $\mathrm{D}_{\mathrm{b}}$ (diâmetro da base a $20 \mathrm{~cm}$ do chão) e $\mathrm{D}_{\text {sup }}$ (diâmetro na altura da primeira bifurcação), medidas em $\mathrm{cm}$. Como instrumento, empregou-se uma suta de $80 \mathrm{~cm}$.

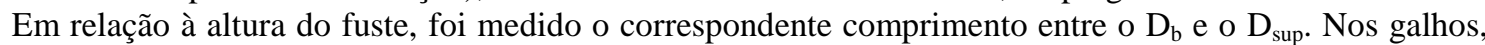
efetuaram-se medidas semelhantes: diâmetro na base do galho, diâmetro da base da bifurcação correspondente e o respectivo comprimento entre esses dois diâmetros.

Para o cálculo de volume real de madeira com casca $(\mathrm{V})$, empregou-se a fórmula de Smalian (LOETSCH et al., 1973), transformando os correspondentes diâmetros em áreas seccionais $\left(\mathrm{A}_{\mathrm{i}}\right)$, e o respectivo comprimento ou altura (h) do fuste ou galho considerado, através da fórmula:

$$
V=\frac{A_{1}+A_{2}}{2} \cdot h
$$

O ajuste do número de indivíduos arbóreos presentes nas parcelas por centro de classe de diâmetro foi realizado por meio do modelo $Y_{j}=\beta_{0} \cdot e^{\beta_{1} \cdot D_{j}}$ (MEYER, 1952), sendo $Y_{j}$ o estimador do número de árvores por hectare na j-ésima classe de $D A P, \beta_{0}$ e $\beta_{l}$ os coeficientes da equação, $D_{j}$ o diâmetro correspondente ao centro da j-ésima classe de $D A P$ e $e$ a constante dos logaritmos neperianos.

Foram testadas 18 equações volumétricas, que estão descritas na tabela 1 . As equações volumétricas de números 1 a 16 foram extraídas de Loetsch et al. (1973), e as de números 17 e 18 dos trabalhos de Scolforo e Silva (1993) e Rezende et al. (2006). Os respectivos coeficientes $\left(\beta_{x}\right)$ foram calculados pelo método "passo a passo" na análise de regressão, realizada pelo programa Statistica 5.0 (STATISTICA, 2005).

Os modelos matemáticos correspondentes às equações volumétricas foram selecionados hierarquicamente, pelo menor valor apresentado do coeficiente de determinação ajustado e do erro padrão da estimativa. $\mathrm{O}$ melhor modelo corresponderá consequentemente àquele que apresentar os menores valores do coeficiente de determinação ajustado e do erro padrão da estimativa.

O coeficiente de determinação ajustado $\left(R_{\text {aj }}^{2}\right)$ foi obtido pela expressão:

$$
R^{2}{ }_{a j}=R^{2}-\left[\frac{K-1}{N-K}\right] \cdot\left(1-R^{2}\right)
$$

em que $\mathrm{K}$ = número de coeficientes da equação e $\mathrm{N}$ = número de observações. 
Tabela 1. Equações volumétricas testadas.

Table 1. Wood volume tested equations.

\begin{tabular}{|c|c|c|}
\hline Equação & $\begin{array}{ll} & \text { Modelo } \\
\end{array}$ & Autor \\
\hline 1 & $v=\beta_{0}+\beta_{1} d^{2}$ & Kopezky-Gehrhardt \\
\hline 2 & $v=\beta_{0}+\beta_{1} d+\beta_{2} d^{2}$ & Dissescu-Meyer \\
\hline 3 & $v=\beta_{0}+\beta_{1} d+\beta_{2} d^{2}$ & Hohenadl-Krenn \\
\hline 4 & $v=\beta_{0} d^{\beta_{1}}$ & Berkhout \\
\hline 5 & $\log v=\beta_{0}+\beta_{1} \log d$ & Hummel \\
\hline 6 & $\log v=\beta_{0}+\beta_{1} \log d+\beta_{2} \frac{1}{d}$ & Brenac \\
\hline 7 & $v=\beta_{0}+\beta_{2} g$ & Hummel \\
\hline 8 & $v=\beta_{0}+\beta_{1} d^{2} h$ & Spurr \\
\hline 9 & $v=\beta_{0} d^{a_{1}} h^{a_{2}}$ & Schumacher-Hall \\
\hline 10 & $v=\frac{d^{2}}{\beta_{0}+\beta_{1} \frac{1}{h_{1}}}$ & Honner \\
\hline 11 & $v=d^{2}\left(\beta_{0}+\beta_{1} h\right)$ & Ogaya \\
\hline 12 & $v=\beta_{0}+\beta_{1} d^{2}+\beta_{2} d^{2} h+\beta_{3} h$ & Stoate \\
\hline 13 & $v=\beta_{1} d^{2}+\beta_{2} d^{2} h+\beta_{3} d h^{2}+\beta_{4} h^{2}$ & Näslund \\
\hline 14 & $v=\frac{d^{2} h}{\beta_{0}+\beta_{1} d}$ & Takata \\
\hline 15 & $\log v=\beta_{0}+\beta_{1} \log \left(d^{2} h\right)$ & Spurr $(\log )$ \\
\hline 16 & $\log v=\beta_{0}+\beta_{1} d+\beta_{2} d^{2}+\beta_{3} d h+\beta_{4} d^{2} h+\beta_{5} h$ & Meyer \\
\hline 17 & $V=\beta_{0}+\beta_{1} \cdot C A P^{2} \cdot H_{t}^{\beta_{2}}$ & Scolforo e Silva (1993) \\
\hline 18 & $V=\beta_{1} \cdot D_{b}^{2}+\beta_{2} \cdot D_{b}^{2} \cdot H_{t}$ & Rezende et al. (2006) \\
\hline
\end{tabular}

O erro padrão da estimativa ( $S y x$ ) foi estimado pela fórmula:

$$
S_{y x}=\sqrt{Q M_{r e s}}
$$

em que $\mathrm{QM}_{\mathrm{res}}=$ Quadrado Médio do resíduo obtido na análise de variância.

Os parâmetros calculados ficaram ordenados em escores hierárquicos (e.h.), sendo atribuído, neste trabalho, o maior valor à equação que apresentar o maior valor do $\mathrm{R}_{\mathrm{aj}}^{2}$, e à equação que tiver $\mathrm{o}$ 
menor $S_{\mathrm{xy}}$. O número de pontos se deu em função do próprio número de equações, elaborando-se uma classificação ponderada que permitiu uma conclusiva e objetiva seleção dos modelos volumétricos (SOARES; MENDONÇA, 2003).

A qualidade da estimação foi calculada posteriormente, conforme apresentada por Prodan et al. (1997):

Erro médio absoluto $(E M)$ :

$$
(E M)=\frac{1}{n} \sum_{i=1}^{n}(v i-\overline{v i})
$$

Erro médio absoluto (EMa) em valor percentual (\%):

$$
(E M a)=\frac{1}{n} \sum_{i=1}^{n}\left[100\left(\frac{v i-\overline{v i}}{\overline{v i}}\right)\right]
$$

Desvio global percentual $(D G)$ :

$$
D G \%=\frac{\sum_{i=1}^{n} v i-\sum_{i=1}^{n} \overline{v i}}{\sum_{i=1}^{n} \overline{v i}} \cdot 100
$$

em que $v i=$ volume individual de cada árvore na amostra, $\overline{v i}=$ volume médio estimado da árvore na parcela e $n=$ número de árvores.

Para a equação selecionada, foi efetuada análise de variância (ANOVA), para se obter o nível de significância da análise de regressão. O valor de p foi calculado pelo programa Statistica 5.0. Gráficos sobre a relação tridimensional do volume, altura e diâmetro também foram realizados por esse programa.

\section{RESULTADOS E DISCUSSÃO}

Um total de 490 árvores com DAP igual ou superior a $5 \mathrm{~cm}$ foram medidas, ficando classificadas em 51 espécies, distribuídas em 28 famílias (Tabela 2).

Observa-se, pelos dados apresentados na tabela 2, que as espécies Ouratea spectabilis e Hymenaea stigonocarpa apresentaram os maiores valores em densidade de indivíduos arbóreos, 50 e 45 $\mathrm{n} / \mathrm{ha}$, respectivamente, correspondendo ambos a $19 \%$ da população amostrada. Dez espécies (2\%) foram catalogadas com apenas 1 indivíduo arbóreo.

Distribuídos os 490 indivíduos arbóreos em classes de diâmetro de $2 \mathrm{~cm}$ (Tabela 3), formou-se uma distribuição diamétrica de 16 classes, concentrando uma frequência de 325 indivíduos (66,33\%) nas primeiras três classes. O correspondente polígono de frequência (Figura 1) mostra uma clara tendência de uma curva do "J" invertido ( $y=168,11 \cdot e^{-0,3435 \cdot x}, \mathrm{R}^{2}=0,85, \mathrm{p}<0,012$ e erro do ajuste $\left.=0,074\right)$. A curva segue o padrão típico para as comunidades vegetais do ecossistema cerrado, conforme apresentado por Amaral et al., 2006; Assunção; Felfili, 2004; Felfili; Silva Júnior, 2001; Felfili et al., 2004, e Ratter et al., 2003. O valor do $\mathrm{R}^{2}$ igual a $85 \%$ permite interpretar, como é mostrado na figura 1 , que a comunidade vegetal estudada teve marcante interferência antrópica, principalmente na sua primeira classe diamétrica e entre as classes de 13 a $19 \mathrm{~cm}$.

O DAP médio aritmético da comunidade vegetal estudada registrou um valor de $10,25 \mathrm{~cm}$. A área basal ocupada foi de $4,92 \mathrm{~m}^{2} / \mathrm{ha}$.

Os resultados da cubagem rigorosa que identificou os parâmetros volumétricos para o fuste e correspondentes galhos estão sintetizados na tabela 2. O cálculo do volume com casca obtido pela cubagem rigorosa forneceu um total de $16,18 \mathrm{~m}^{3} / \mathrm{ha}$ de madeira, correspondendo a $10,84 \mathrm{~m} / \mathrm{ha}(67 \%) \mathrm{o}$ volume total dos fustes e a $5,33 \mathrm{~m} 3 / \mathrm{ha}(33 \%)$ o dos galhos. Batalha et al. (2004) encontraram para o cerrado s.s. da região Sudeste (São Paulo) um volume de madeira igual a $16,479 \mathrm{~m}^{3} / \mathrm{ha}$. Rezende et al. (2006) registraram para um cerrado s.s. localizado no Distrito Federal um valor igual a 25,10 $\mathrm{m}^{3} / \mathrm{ha}$. 
Tabela 2. Espécies arbóreas e correspondente volume de madeira $\mathrm{em} \mathrm{m}^{3}$ em um hectare de cerrado stricto sensu.

Table 2. Tree species and wood volume in $\mathrm{m}^{3}$ from one hectare of the "cerrado stricto sensu".

\begin{tabular}{|c|c|c|c|c|c|}
\hline Espécie & Família & $\mathbf{n} / \mathbf{h a}$ & $\begin{array}{c}\begin{array}{c}\text { Volume fuste } \\
\left(\mathbf{m}^{3} / \mathbf{h a}\right)\end{array} \\
\end{array}$ & $\begin{array}{c}\text { Volume galhos } \\
\left(\mathrm{m}^{3} / \mathbf{h a}\right)\end{array}$ & $\begin{array}{c}\text { Volume total } \\
\left(\mathbf{m}^{3} / \mathbf{h a}\right)\end{array}$ \\
\hline Agonandra brasiliensis Miers. & Opiliaceae & 2 & 0,01481 & & 0,01481 \\
\hline Annona crassiflora Mart. & Annonaceae & 4 & 0,11779 & 0,01094 & 0,12873 \\
\hline Aspidosperma macrocarpon Mart. & Apocynaceae & 10 & 0,09341 & 0,01548 & 0,10890 \\
\hline Aspidosperma tomentosum Mart. & Apocynaceae & 17 & 0,23622 & 0,03269 & 0,26891 \\
\hline Austroplenckia populnea (Reiss.) Lund & Celastraceae & 3 & 0,06919 & 0,00382 & 0,07302 \\
\hline Bowdichia virgilioides H.B.K. & Leguminosae & 10 & 0,55918 & 0,17002 & 0,72920 \\
\hline Byrsonima correifolia A. Juss. & Malpighiaceae & 14 & 0,28930 & 0,10443 & 0,39374 \\
\hline Byrsonima crassa Nied. & Malpighiaceae & 10 & 0,10620 & 0,02344 & 0,12965 \\
\hline Byrsonima crassifolia (L.) H. B. K. & Malpighiaceae & 1 & 0,00832 & 0,00292 & 0,01125 \\
\hline Connarus suberosus Planch. var. suberosus & Connaraceae & 4 & 0,10182 & 0,00510 & 0,10693 \\
\hline Caryocar brasiliense Camb. & Caryocaraceae & 14 & 0,81468 & 1,33189 & 2,14658 \\
\hline Chamaecrista imbricans (I. \& B.) I. \& B. & Leguminosae & 9 & 0,07496 & 0,00815 & 0,08312 \\
\hline Dalbergia miscolobium Benth. & Leguminosae & 2 & 0,17302 & 0,04207 & 0,21510 \\
\hline Dimorphandra mollis Benth. & Leguminosae & 10 & 0,07358 & & 0,07358 \\
\hline Eriotheca pubescens Schott. \& Ende & Bombacaceae & 1 & 0,08943 & 0,00656 & 0,09600 \\
\hline Erytroxylum amplifolium O. E. Sch. & Erythroxylaceae & 1 & 0,00832 & & 0,00832 \\
\hline Eugenia dysenterica DC. & Myrtaceae & 9 & 0,00435 & 0,00146 & 0,00582 \\
\hline Guapira noxia (Netto) Lund & Nyctaginaceae & 18 & 0,16895 & 0,02820 & 0,19716 \\
\hline Hymenaea stigonocarpa Mart. ex Hayne & Leguminosae & 45 & 0,67806 & 0,27784 & 0,95591 \\
\hline Kielmeyera coriacea (Spreng.) Mart. \& Zucc. & Guttiferae & 3 & 1,25238 & 0,65758 & 1,90997 \\
\hline Lafoensia pacari St.-Hil. & Lythraceae & 1 & 0,06169 & & 0,06169 \\
\hline Machaerium opacum Vog. & Leguminosae & 32 & 0,01786 & 0,00479 & 0,02266 \\
\hline Miconia burchellii Triana & Melastomataceae & 8 & 0,44490 & & 0,56395 \\
\hline Miconia cuspidata Naud. & Melastomataceae & 3 & 0,20396 & 0,09808 & 0,30204 \\
\hline Miconia ferruginata DC. & Melastomataceae & 1 & 0,07613 & 0,04422 & 0,12035 \\
\hline Miconia ferruginea DC. var. latifolia DC. & Melastomataceae & 3 & 0,01048 & 0,00588 & 0,01636 \\
\hline Miconia fallax DC. & Melastomataceae & 1 & 0,08075 & 0,08665 & 0,16740 \\
\hline Myrcia fallax (Rich.) DC. & Myrtaceae & 1 & 0,00907 & & 0,00907 \\
\hline Myrsine guianensis (Aubl.) Kuntze & Myrsinaceae & 4 & 0,01472 & 0,00365 & 0,01838 \\
\hline Neea theifera Oerst. & Nyctaginaceae & 26 & 0,03926 & & 0,03926 \\
\hline Ocotea spixiana (Ness) Mez. & Lauraceae & 1 & 0,38415 & 0,10818 & 0,49234 \\
\hline Ouratea spectabilis (Mart.) Engl. & Ochnaceae & 50 & 0,02945 & & 0,02945 \\
\hline Palicourea rígida Kunth & Rubiaceae & 3 & 0,35194 & 0,11668 & 0,46863 \\
\hline Piptocarpha rotundifolia (Less.) Baker & Asteraceae & 5 & 0,01728 & & 0,01728 \\
\hline Pouteria ramiflora (Mart.) Radlk. & Sapotaceae & 33 & 0,05657 & & 0,05657 \\
\hline Pterodon polygaliflorus Benth. & Leguminosae & 10 & 0,71735 & 0,22723 & 0,94459 \\
\hline Qualea grandiflora Mart. & Vochysiaceae & 7 & 0,61382 & 0,74649 & 1,36031 \\
\hline Qualea parviflora Mart. & Vochysiaceae & 36 & 0,13232 & 0,04064 & 0,17296 \\
\hline Roupala montana Aubl. & Proteaceae & 1 & 1,02028 & 0,56854 & 1,58883 \\
\hline Salacia crassifolia (Mart.) G. Don & Hippocrateraceae & 8 & 0,00781 & & 0,00781 \\
\hline Salvertia convallariodora St.-Hil. & Vochysiaceae & 2 & 0,07061 & 0,01539 & 0,08600 \\
\hline Schefflera macrocarpa (Seem.) D.C. Frodin & Araliaceae & 5 & 0,03298 & 0,00761 & 0,04060 \\
\hline Sclerolobium paniculatum Vog. & Leguminosae & 18 & 0,74193 & 0,24549 & 0,98743 \\
\hline Stryphnodendron adstringens (Mart.) Coville & Leguminosae & 3 & 0,03146 & 0,00511 & 0,03658 \\
\hline Strychnos pseudoquina St.-Hil. & Loganiaceae & 14 & 0,34542 & 0,08543 & 0,43086 \\
\hline Styrax camporum Pohl. & Styracaceae & 6 & 0,08014 & 0,00408 & 0,08422 \\
\hline
\end{tabular}




\begin{tabular}{lccccc}
\hline Tabebuia ochracea Cham. & Bignoniaceae & 1 & 0,00629 & & 0,00629 \\
Vatairea macrocarpa (Benth.) Ducke & Leguminosae & 4 & 0,07164 & 0,00539 & 0,07704 \\
Vochysia elliptica Mart. & Vochysiaceae & 3 & 0,02830 & 0,00958 & 0,03788 \\
Vochysia rufa Mart. & Vochysiaceae & 11 & 0,17235 & 0,03832 & 0,21068 \\
Vochysia thyrsoidea Pohl. & Vochysiaceae & 2 & 0,04769 & 0,02226 & 0,06996 \\
\hline Total & & 490 & 10,85281 & 5,33155 & 16,18436 \\
\hline
\end{tabular}

Tabela 3. Distribuição dos DAPs por classes diamétricas.

Table 3. Diameter distribution.

\begin{tabular}{lcccc}
\hline $\begin{array}{l}\text { Classe } \\
\text { diamétrica }\end{array}$ & $\begin{array}{c}\text { PMC } \\
\text { cm }\end{array}$ & $\mathbf{n}$ & \% & n acumulado \\
\hline $5-7$ & 6 & 68 & 13,88 & 68 \\
$7-9$ & 8 & 151 & 30,82 & 219 \\
$9-11$ & 10 & 106 & 21,63 & 325 \\
$11-13$ & 12 & 68 & 13,88 & 393 \\
$13-15$ & 14 & 29 & 5,92 & 422 \\
$15-17$ & 16 & 29 & 5,92 & 451 \\
$17-19$ & 18 & 11 & 2,24 & 462 \\
$19-21$ & 20 & 8 & 1,63 & 470 \\
$21-23$ & 22 & 4 & 0,82 & 474 \\
$23-25$ & 24 & 4 & 0,82 & 478 \\
$25-27$ & 26 & 3 & 0,61 & 481 \\
$27-29$ & 28 & 1 & 0,2 & 482 \\
$29-31$ & 30 & 4 & 0,82 & 486 \\
$31-33$ & 32 & 1 & 0,2 & 487 \\
$33-35$ & 34 & 1 & 0,2 & 488 \\
$35-37$ & 36 & 2 & 0,41 & 490 \\
\hline
\end{tabular}

PMC: ponto médio da classe diamétrica; n: número de indivíduos.

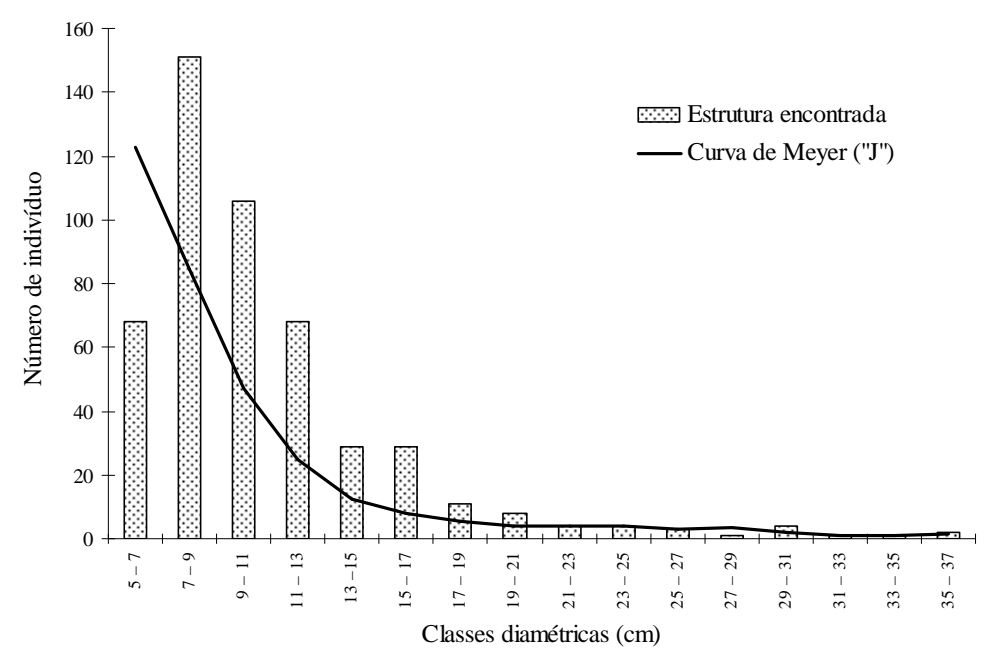

Figura 1. Curva de forma exponencial negativa da distribuição diamétrica de 490 árvores encontradas em um hectare de cerrado stricto sensu, na região de Planaltina de Goiás.

Figure 1. Negative exponential curve of the diametric distribution of 490 savanna tress located in one hectare in the Planaltina region, Goiás state. 
As espécies que apresentaram maior volume de madeira foram: Caryocar brasiliense $(2,14$ $\mathrm{m}^{3} / \mathrm{ha}$, com 14 indivíduos), Kielmeyera coriacea $\left(1,91 \mathrm{~m}^{3} / \mathrm{ha}\right.$, com 3 indivíduos), Roupala montana $(1,59$ $\mathrm{m}^{3} / \mathrm{ha}$, com somente 1 indivíduo) e Qualea grandiflora $\left(1,36 \mathrm{~m}^{3} / \mathrm{ha}\right.$, com 7 indivíduos arbóreos). Essas 4 espécies, com 25 indivíduos arbóreos (5\% da população), perfizeram $43 \%$ do volume total. As espécies Caryocar brasiliense, Miconia fallax e Qualea grandiflora apresentaram maior volume de madeira nos galhos do que no fuste. 12 espécies (23\%) não apresentaram volume dos galhos, uma vez que os respectivos galhos não possuíam o diâmetro mínimo de $5 \mathrm{~cm}$.

Das 18 equações testadas, a que teve melhor ajuste foi a equação de Rezende et al. (2006), por possuir maior significância do coeficiente de determinação ajustado, $\mathrm{R}_{\text {aj }}^{2}>0,99$ (Tabela 4), e menores erros e desvios estimados $\left(\mathrm{S}_{\mathrm{xy}}=10,22 \% ; \mathrm{EM}=0,21 ; \mathrm{EMa}=7,82\right.$ e $\left.\mathrm{DG}=5,32\right)$. Todos os coeficientes dessa equação foram significativos, sendo ela descrita como: $v=12,1147 \cdot 10^{-5} \cdot D_{b}^{2}+16,1578 \cdot 10^{-6} \cdot D_{b}{ }^{2} \cdot H_{t}$.

Tabela 4. Resultado da análise de regressão do ajustes das equações pelo método passo a passo.

Table 4. Results of the regression analysis of the adjusted equations by the step by step method.

\begin{tabular}{|c|c|c|c|c|c|c|c|c|c|c|c|}
\hline \multirow{2}{*}{ Equação } & \multicolumn{6}{|c|}{ Coeficientes } & \multirow{2}{*}{$\mathbf{R}_{\text {aj }}^{2}$} & \multirow{2}{*}{$\mathrm{S}_{\mathrm{xy}}(\%)$} & \multirow{2}{*}{ EM } & \multirow{2}{*}{ EMa } & \multirow{2}{*}{ DG } \\
\hline & $\beta_{0}$ & $\beta_{1}$ & $\beta_{2}$ & $\beta_{3}$ & $\mathrm{~B}_{4}$ & $\beta_{5}$ & & & & & \\
\hline Kopezky-Gehrhardt & 0,98420 & $0,04598 *$ & - & - & - & - & 0,89 & 17,05 & 0,452 & 9,840 & 8,048 \\
\hline Dissescu-Meyer & $0,05780^{*}$ & 1,26590 & $0,69870 *$ & - & - & - & 0,85 & 12,38 & 0,546 & 6,881 & 7,961 \\
\hline Hohenadl-Krenn & $-0,08147$ & $-0,00529^{*}$ & $0,00132 *$ & - & - & - & 0,93 & 13,95 & 0,334 & 11,597 & 7,205 \\
\hline Berkhout & 0,02370 & $2,36540^{*}$ & - & - & - & - & 0,94 & 15,66 & 0,383 & 8,671 & 9,084 \\
\hline Hummel & 0,56730 & $0,01250^{*}$ & - & - & - & - & 0,88 & 19,16 & 0,462 & 9,395 & 11,199 \\
\hline Brenac & $0,01375^{*}$ & $0,00124^{*}$ & $1,24440 *$ & - & - & - & 0,88 & 18,20 & 0,517 & 10,569 & 8,667 \\
\hline Hummel & $0,75300^{*}$ & - & $0,00080^{*}$ & - & - & - & 0,83 & 13,79 & 0,546 & 9,213 & 10,169 \\
\hline Spurr & 0,04900 & $0,00004 *$ & - & - & - & - & 0,90 & 19,40 & 0,385 & 11,700 & 7,606 \\
\hline Schumacher-Hall & $-9,85400^{*}$ & $1,25980^{*}$ & $1,36520^{*}$ & - & - & - & 0,93 & 16,61 & 0,657 & 8,330 & 10,329 \\
\hline Honner & $3,12400^{*}$ & $0,10055^{*}$ & - & - & - & - & 0,85 & 25,68 & 0,549 & 8,543 & 6,750 \\
\hline Ogaya & 0,00235 & $0,11190^{*}$ & - & - & - & - & 0,87 & 15,33 & 0,610 & 7,991 & 6,259 \\
\hline Stoate & $-0,15170^{*}$ & 0,00002 & $0,00003 *$ & 0,01201 & - & - & 0,84 & 13,09 & 0,574 & 8,708 & 7,282 \\
\hline Näslund & - & $0,00007^{*}$ & 0,00004 & 0,00001 & $0,00006^{*}$ & - & 0,84 & 20,71 & 0,585 & 7,633 & 7,842 \\
\hline Takata & 0,07420 & $0,04990^{*}$ & - & - & - & - & 0,86 & 25,21 & 0,658 & 8,734 & 4,943 \\
\hline Spurr (log) & 0,00011 & $0,00002 *$ & - & - & - & - & 0,87 & 13,11 & 0,548 & 10,230 & 7,921 \\
\hline Meyer & $-0,03456^{*}$ & $-0,00200^{*}$ & 0,00001 & $0,00054^{*}$ & 0,00004 & $0,00195^{*}$ & 0,83 & 14,40 & 0,334 & 10,126 & 7,662 \\
\hline Scolforo e Silva (1993) & $0,21450^{*}$ & $0,00025^{*}$ & $0,01590 *$ & - & - & - & 0,98 & 11,33 & 0,338 & 8,130 & 5,733 \\
\hline Rezende et al. (2006) & - & $12,11400^{*}$ & $16,15700^{*}$ & - & - & - & 0,99 & 10,22 & 0,214 & 7,828 & 5,324 \\
\hline
\end{tabular}

* Coeficientes significativos utilizados na equação.

A análise de variância mostrou a qualidade do ajuste para a equação escolhida, apresentando uma alta significância estatística entre as variáveis independentes $\left(D_{b}^{2}\right.$ e $\left.D_{b}^{2} \cdot H_{t}\right)$ e a variável dependente $(v)$. Consequentemente, o valor de $\mathrm{F}$ calculado foi maior que o tabelado.

A análise gráfica dos resíduos (EMa) (Figura 2) mostrou uma coerente distribuição da nuvem dos pontos, que confirmam o menor erro para a equação proposta por Rezende et al. (2006), em ambos os lados da linha zero, e mostram a normalidade e homogeneidade da variação entre os dados reais e os estimados para a interpretação da análise de regressão, corroborando os trabalhos de Silva et al. (2006).

Rezende et al. (2006), com estudos sobre equações para ajustes de volume em áreas de cerrado s.s., observaram a variabilidade natural existente na estrutura da vegetação e nas formas dos troncos das espécies lenhosas desse bioma, ocasionando um maior número de outliers nos gráficos de volume e consequentemente influenciando o erro médio absoluto. Para esses autores, erros entre 15 e $30 \%$ em ajustes dos dados de diâmetro e altura dos indivíduos arbóreos para volume e equações regionalizadas podem ser consideradas plenamente aceitáveis, porém ajustes como o deste trabalho demonstraram que menores erros médios absolutos podem ser obtidos, com erros de 10 a $20 \%$.

Quanto à relação das variáveis de volume $\left(\mathrm{m}^{3}\right)$ real (fórmula de Smalian) e o estimado (equação ajustada) com a altura total e o diâmetro da base das árvores (Figura 3), observa-se uma concentração dos valores de volume $\left(\mathrm{m}^{3}\right)$ entre 0,0160 e 0,0186, ou seja, 97,15\% das árvores de cerrado s.s. estariam 
enquadradas nesse agrupamento. Delitti et al. (2006) e Abdala et al. (1998), com dados de biomassa arbórea individual em cerrado s.s., corroboram essa concentração, descrevendo que os indivíduos arbóreos possuem concentrações de biomassa bem próximas.
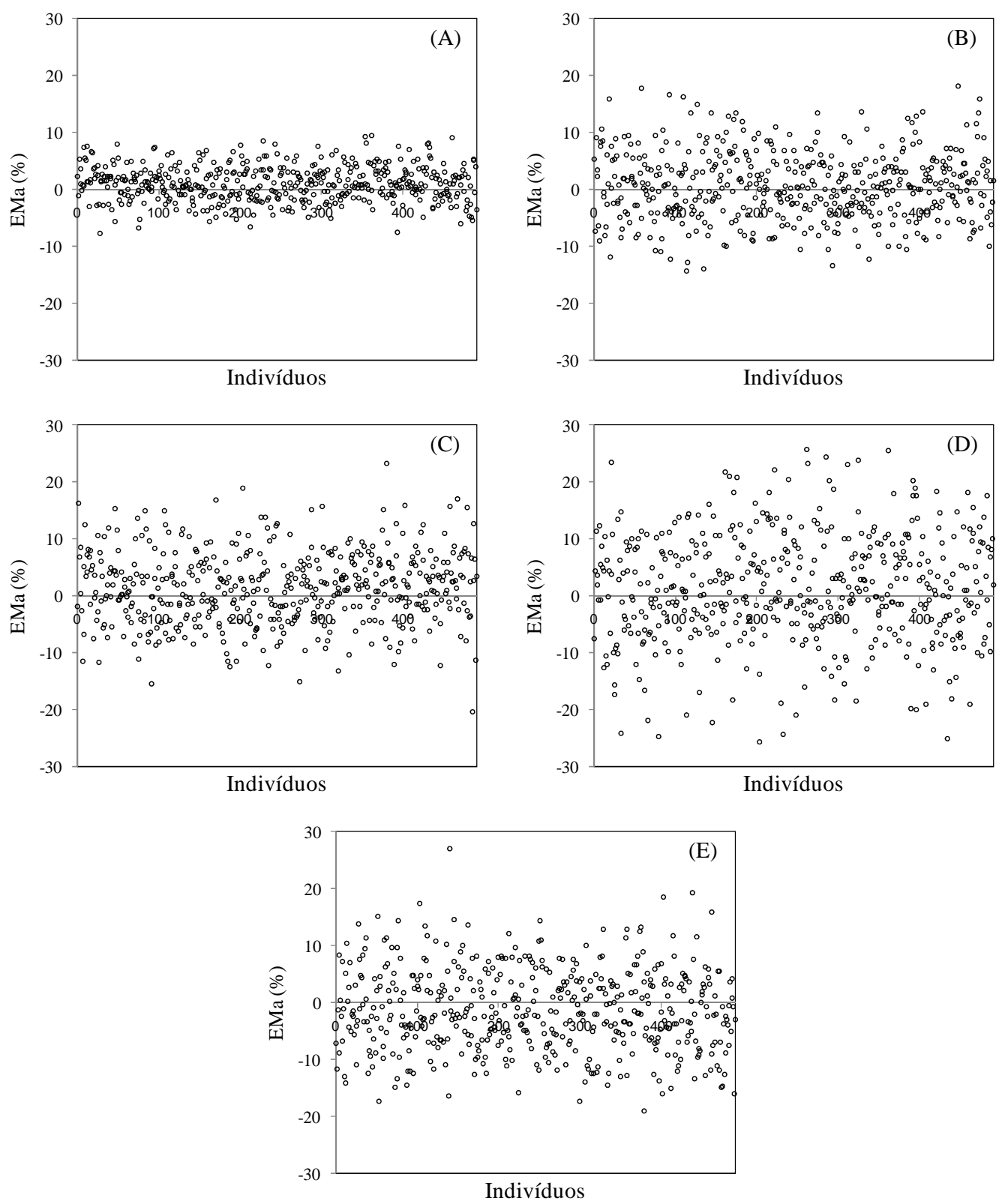

Figura 2. Erro médio absoluto (EMa) em valor percentual (\%) das equações: (A) Rezende et al. (2006), (B) Scolforo; Silva (1993), (C) Berkhout, (D) Schumacher-Hall, (E) Hohenadl-Krenn.

Figure 2. Mean Absolute Error (EMa) in percentual value (\%) of the equations: (A) Rezende et al. (2006), (B) Scolforo; Silva (1993), (C) Berkhout, (D) Schumacher-Hall, (E) Hohenadl-Krenn. 


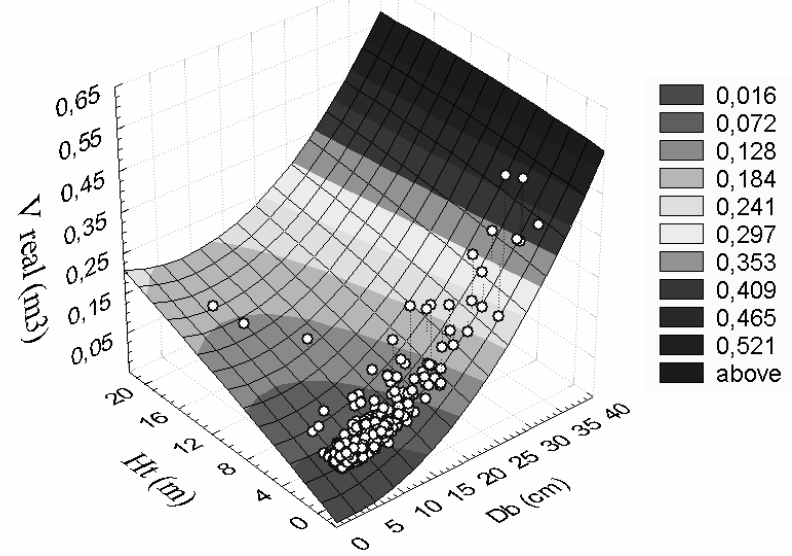

(A)

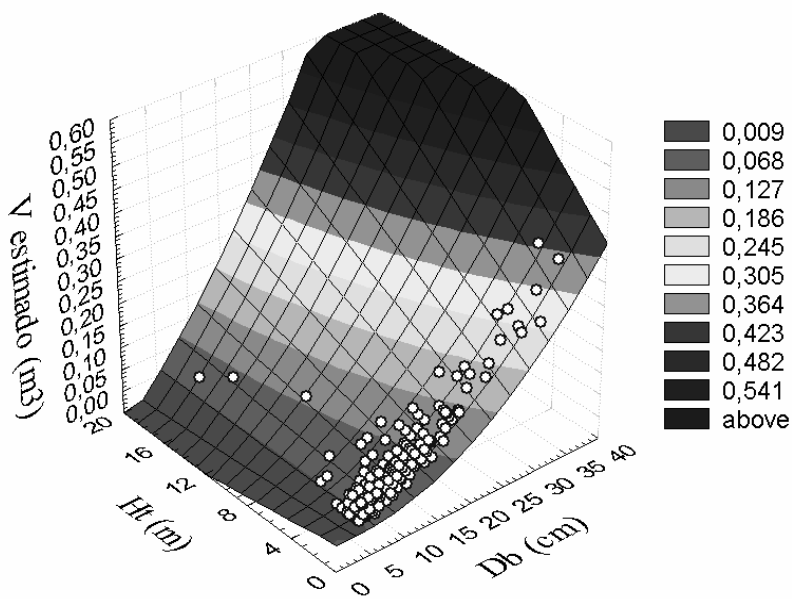

(B)

Figura 3. Relação das variáveis de volume $\left(\mathrm{m}^{3}\right)$ real (A - equação de Smalian) e estimado (B - equação ajustada) com a altura total ( $\mathrm{Ht}$ ) e o diâmetro da base ( $\mathrm{Db}$ ) de árvores de cerrado em Planaltina de Goiás.

Figure 3. Relationship between the variables real (A - Smalian equation) and estimated (B - adjusted equation) volume $\left(\mathrm{m}^{3}\right)$, with total height $(\mathrm{Ht})$ and diameter on the base tree $(\mathrm{Db})$ of the "cerrado" trees in Planaltina area, Goiás state.

\section{CONCLUSÕES}

A riqueza florística mostrou a existência de 51 espécies arbóreas.

O cálculo de volume de madeira com casca, através da fórmula de Smalian, identificou um total de $16,1843 \mathrm{~m}^{3} / \mathrm{h}$, correspondendo a $10,8528 \mathrm{~m}^{3} / \mathrm{ha}$ do volume dos fustes e $5,3315 \mathrm{~m}^{3} / \mathrm{ha}$ de madeira dos galhos. As espécies que apresentaram os maiores volumes de madeira foram: Caryocar brasiliense, Kielmeyera coriacea e Roupala montana.

O modelo volumétrico que melhor ficou ajustado aos dados coletados foi o de Rezende et al., que apresentou o maior $\mathbf{R}_{\text {aj }}^{2}(0,99)$ e o menor desvio padrão da estimativa $\left(S_{x y}=10,22 \%\right)$.

\section{REFERÊNCIAS}

ABDALA, G. C., CALDAS, L. S., HARIDASAN, M.; EITEN, G. Above and belowground organic matter and root:shoot ratio in a cerrado in Central Brazil. Brazilian Journal of Ecology, local, v. 2, p. 11-23, 1998. 
AMARAL, A. G.; PEREIRA, F. F. O.; MUNHOZ, C. B. R. Fitossociologia de uma área de cerrado rupestre na Fazenda Sucupira, Brasília, DF. Cerne, Lavras, v. 12, n. 4, p. 350-359, 2006.

ASSUNÇÃO, S. L.; FELFILI, J. M. Fitossociologia de um fragmento de cerrado sensu stricto na APA do Paranoá, DF, Brasil. Acta Botanica Brasilica, São Paulo, v. 18, n. 4, p. 903-909, 2004.

BATALHA, M. A.; MARTINS, F. R. Floristic, frequency, and vegetation life-form spectra of a cerrado site. Brazilian Journal of Biology, São Carlos, v. 64, n. 2, p. 201-209, 2004.

DELITTI, W. B. C.; MEGURO, M.; PAUSAS, J. G. Biomass and mineralmass estimates in a "cerrado" ecosystem. Revista Brasileira de Botânica, São Paulo, v. 29, n. 4, p. 531-540, 2006.

FELFILI, J. M.; SILVA JUNIOR, M. C. da. (Orgs.) Biogeografia do Bioma Cerrado: estudo fitofisionômico da Chapada do Espigão Mestre do São Francisco. Brasília, DF: Universidade de Brasília, 2001. 152 p.

FELFILI, J. M.; SILVA JUNIOR, M. C.; SEVILHA, A. C.; FAGG, C. W.; WALTER, B. M. T.; NOGUEIRA P. E.; REZENDE, A.V. Diversity, floristic and structural patterns of cerrado vegetation in Central Brazil. Plant Ecology, Ames, n. 174, p. 37-46, 2004.

FONSECA, M. S.; SILVA JÚNIOR, M. C. Fitossociologia e similaridade florística entre trechos de cerrado sentido restricto em interflúvio e em vale no Jardim Botânico de Brasília, DF. Acta Botanica Brasilica, São Paulo, v. 18, n. 1, p. 19-29, 2004.

IMAÑA-ENCINAS, J.; PAULA, J. E. de. Análise da vegetação de cerrado no município de Santa Quitéria - Maranhão. Brasil Florestal, Brasília, DF, n. 78, p. 33-42, 2003.

LOETSCH, F.; ZÖHRER, F.; HALLER, K. E. Forest inventory. Munich: BLV, 1973. v.2. 469p.

MEYER, H. A. Structure, growth and drain in balanced uneven-aged forests. Journal of Forestry, Washington, DC, v. 50, p. 85-92, 1952.

OLIVEIRA, A. D.; LEITE, A. P.; BOTELHO, S. A.; SCOLFORO, J. R. S. Avaliação econômica da vegetação de cerrado submetida a diferentes regimes de manejo e de povoamentos de Eucaliptos plantado em monocultivo. Cerne, Lavras, v. 4, n. 1, p. 34-56, 1998.

PAULA, J. E. de; IMAÑA-ENCINAS, J.; SUGIMOTO, N. Levantamento quantitativo em três hectares de vegetação de cerrado. Pesquisa Agropecuária Brasileira, Brasília, DF, v. 33, n. 5, p. 613-620, 1998

PRODAN, M.; ROLAND, P.; COX, F.; REAL, P. Mensura Forestal. San José, Costa Rica: IICA, 1997. 586 p. (Serie de investigación y educación en desarrollo sostenible).

RATTER, J. A.; BRIDGEWATER, S.; RIBEIRO, J. F. Analysis of the floristic composition of the Brazilian cerrado vegetation III: comparison of the wood vegetation. Edinburgh Journal of Botany, Edinburgh, n. 60, p. 57-109, 2003.

REZENDE, A. V.; VAlE, A. T.; SANQUETTA, C. R.; FIGUEIREDO FILHO, A.; FELFILI, J. M. Comparação de modelos matemáticos para estimativa do volume, biomassa e estoque de carbono da vegetação lenhosa de um cerrado sensu stricto em Brasília, DF. Scientia Forestalis, Piracicaba, n. 71, p. 65-76, 2006.

SILVA, L. M. S.; RODRIGUEZ, L. C. E.; CAIXETA-FILHO, J. V.; BAUCH, S. C. Fitting a taper function to minimize the sum of absolute deviations. Scientia Agricola, Piracicaba, v. 63, n. 5, p. 460470, 2006.

SCOLFORO, J. R. S.; SILVA, S. T. O conceito de "floresta balanceada de Meyer" como opção para intervenção em cerrado senso stricto. In: CONGRESSO FLORESTAL BRASILEIRO, 7., 1993, Curitiba. Anais... Curitiba: SBS, 1993. v. 1, p. 378-381.

SOARES, T. M.; MENDONÇA, M. C. M. Construção de um modelo de regressão hierárquico para os dados do SIMAVE-2000. Pesquisa Operacional, Rio de Janeiro, v. 23, n. 3, p. 421-441, 2003.

STATISTICA for Windows: general conventions and statistics. Tulsa : StatSoft, 1995. v. 1. 